\title{
The Neutrophil-Lymphocyte Ratio Is an Independent Prognostic Factor for Overall Survival in Hispanic Patients with Gastric Adenocarcinoma
}

\author{
A. Ramos-Esquivel ${ }^{1}$ (D) - E. Cordero-García ${ }^{2}$ - D. Brenes-Redondo ${ }^{3}$ - W. Alpízar-Alpízar ${ }^{4}$
}

(C) Springer Science+Business Media, LLC, part of Springer Nature 2018

\begin{abstract}
Introduction High values of neutrophil-lymphocyte ratio (NLR) and platelet-lymphocyte ratio (PLR) are related with poor prognosis in patients with gastric cancer. However, this association has been rarely assessed in Hispanic populations that show important clinicopathological differences to Asian and Caucasian patients. In this study, we determined the prognostic value of these biomarkers in Hispanic patients from Costa Rica.

Materials and Methods We retrieved data regarding pre-treatment NLR and PLR, as well as clinical variables from medical records of 381 consecutive gastric cancer patients treated in four major hospitals in Costa Rica between 2009 and 2012. Univariate and multiple Cox regression analyses were performed to assess the value of NLR and PLR as predictors of overall survival (OS) and disease-free survival (DFS). The best cutoff point was based on the maximization of the Log-rank test.

Results Median follow-up was 13.21 months. In univariate analysis, a NLR $\geq 5$ was associated with reduced DFS (hazard ratio (HR) 2.31 ; 95\% confidence interval (CI) $1.78-3.00 ; p<0.001$ ) and poor OS (HR 2.24; 95\% CI 1.72-2.92; $p<0.001$ ). Similarly, a PLR $\geq 350$ was associated with worse DFS (HR 2.28; 95\% CI 1.70-3.06; $p<0.001$ ) and poor OS (HR 2.33; 95\% CI 1.73-3.13; $p<0.001)$. After adjustment for potential confounders, multivariate analysis revealed that only the NLR $\geq 5$ was independently associated with worse DFS (HR 1.97; 95\% CI 1.44-2.47) and OS (HR 1.59; 95\%CI 1.15-2.28).

Conclusions NLR $\geq 5$ was independently associated with worse OS and DFS in Hispanic patients with gastric cancer.
\end{abstract}

Keywords Gastric cancer $\cdot$ Lymphocyte $\cdot$ Neutrophil $\cdot$ Platelet $\cdot$ Prognosis

\section{Introduction}

Gastric cancer represents the fifth most common form of cancer and the third cause of cancer-related death in both

The abstract of this manuscript was presented at the ESMO XX World Congress on Gastrointestinal Cancer 2018, Barcelona, Spain.

A. Ramos-Esquivel

allan.ramos@ucr.ac.cr

1 Departamento de Farmacología, Escuela de Medicina, Universidad de Costa Rica, Sede Rodrigo Facio, San Pedro, PO BOX 2082, San José, Costa Rica

2 Instituto de Investigaciones Farmacéuticas, Facultad de Farmacia, Universidad de Costa Rica, San Pedro, Costa Rica

3 Departamento de Oncología Médica, Caja Costarricense de Seguro Social, Hospital Max Peralta, Cartago, Costa Rica

4 Departamento de Bioquímica, Escuela de Medicina and Centro de Investigación en Estructuras Microscópicas (CIEMIC), Universidad de Costa Rica, San Pedro, Costa Rica sexes worldwide [1]. Almost $70 \%$ of cases occur in developing countries, particularly in Eastern Asia, Central and Eastern Europe, and Central and South America. In these regions, stomach cancer is the third most frequently diagnosed malignancy among men and the fifth cause of incidence among women [2]. In 2015, stomach cancer was the second cause of cancer-related mortality in both sexes among Costa Rican patients [3]. This in line with the mortality rates reported in other Central and South American countries, which are among the highest in the world $[4,5]$. Despite the high prevalence and mortality of this disease in Hispanic countries, patients from this region are usually under-represented in clinical trials, and few data are available regarding prognostic factors for overall survival (OS) and disease-free survival (DFS).

Previous studies have shown a different clinicopathologic behavior of this malignancy among Hispanic patients [6, 7] that may be partly related to their genetic background. These studies have shown that gastric adenocarcinoma in Hispanic populations are more likely to affect young patients with 
diffuse and undifferentiated tumors of distal location [6, 7]. Other reports have consistently revealed shorter overall survival and poorer long-term clinical outcomes in Hispanic patients than in White-non-Hispanic populations [8, 9].

The neutrophil-lymphocyte ratio (NLR) and the plateletlymphocyte ratio (PLR) have been recently identified as prognostic factors in patients with solid tumors, including gastric cancer $[10,11]$. However, the prognostic role of these biomarkers in patients with gastric adenocarcinoma from Hispanic origin is largely unknown. Given the fairly distinctive outcomes and clinical characteristics of this malignancy in this population, we performed the present study to determine the prognostic value of the NLR and PLR as predictors of OS and DFS in patients with gastric cancer from a Hispanic background (Costa Rica), and to establish the best cutoff value to adequately classify patients according to their baseline risk.

\section{Methods}

The clinical records of all consecutive patients diagnosed with gastric adenocarcinoma from January 2009 to January 2012 in four major public hospitals of Costa Rica (Caja Costarricense de Seguro Social) were retrospectively reviewed. Baseline clinical and tumor characteristics were manually retrieved from the clinical records. The NLR (defined as the absolute neutrophil count divided by the absolute lymphocyte count) and PLR (defined as the absolute platelet count divided by the absolute lymphocyte count) were calculated from full blood test performed prior surgery or chemotherapy. Patients with hematological disorders, corticosteroids, or any kind of acute or chronicle inflammatory diseases were excluded, as well as patients with no pre-treatment blood tests. All cases were classified according to the TNM criteria as described by 7th Edition of the American Joint Committee on Cancer. Patients with non-metastatic gastric cancer underwent a surgical procedure that consisted of total or subtotal gastrectomy with lymph node dissection (D1 or D2) depending on tumor location and surgeon's preference.

Patients could receive adjuvant treatment, either chemotherapy or chemoradiotherapy (CRT), according to the medical oncologist consultation. In case of metastatic disease and good performance status (ECOG 0 to 2), patients received cytotoxic therapy.

Patients were followed up at least at 3-month intervals during the first 2 years, then at 6-month intervals for 3 years, and yearly thereafter. Follow-up included a computer tomography scan and esophagogastroduodenoscopic examination as clinically indicated.

The primary outcome of this study was OS as defined from the date of first cancer treatment to the date of death according to the Costa Rican National Registry. DFS was defined from the date of primary surgery to the date of clinically confirmed recurrence or death. Recurrence was defined as the presence of a biopsy-proven tumor with adenocarcinoma features or the presence of imaging highly suspicious of tumor recurrence.

\section{Statistical Analysis}

Categorical variables are presented as percentages and continuous variables as means and standard deviations (SD).

The best NLR and PLR cutoff points were determined based on the maximization of the log-likelihood ratio method as proposed by Contal and O'Quigley [12].

The chi-square test or Fisher's exact test were used for comparison of frequencies, while the Student's $t$ test was used for comparison of quantitative variables. An OS and DFS analysis was performed using the Kaplan-Meier method. The Log-rank test was used to compare survival curves. Univariate and multiple COX regression analyses were used to calculate the crude and adjusted hazard ratios (HR) with their $95 \%$ confidence interval $(95 \% \mathrm{CI})$. Variables with a $p$ value less than 0.10 by univariate analysis were included in the multivariate analysis using the backward stepwise technique. An interaction term (NLR*PLR) was added to the model to test for the interactive effect of NLR and PLR.

A $p$ value of less than 0.05 was considered statistically significant. Statistical analysis was carried out using SPSS version 21.0 for Mac (Chicago, Illinois, USA). All statistical tests were two-tailed.

\section{Results}

A total of 381 patients met the inclusion criteria. Table 1 summarizes the main characteristics of the included patients and compares baseline characteristics according to the NLR and PLR. After statistical analysis, the optimal cutoff was set at 5 for NLR (corresponding to a log-rank value of 37.15) and 350 for PLR (corresponding to a logrank value of 33.32). Median NLR and PLR were 2.61 and 180.72 , respectively. Patients allocated to the high NLR and PLR groups were more likely to have an advanced clinical stage, poor performance status, and did receive less chemotherapy or surgery in comparison with patients with low NLR and PLR values.

Median follow-up time was 13.21 months (range 084 months). During this period, a total of 273 patients $(71.7 \%)$ died. Overall, 5-year survival probability was $27.1 \%$ (95\% CI 22.61-31.62). Figure 1 shows the OS probability according to NLR and PLR categories. Median survival time was 5.0 months (95\% CI 1.58 8.42) for patients with NLR $\geq 5$ and 18.2 months $(95 \% \mathrm{CI}$ 12.10-24.32) for patients with NLR less than 5 (HR 2.24; 95\% CI 1.72-2.92, Log-rank test: $p<0.001)$. Similarly, 
Table 1 General characteristics of the included population

\begin{tabular}{|c|c|c|c|c|c|c|c|}
\hline \multirow[t]{2}{*}{ Variables } & \multirow[t]{2}{*}{ All patients $(N=381)$} & \multicolumn{2}{|c|}{ Neutrophil-lymphocyte ratio } & \multirow[t]{2}{*}{$p$ value } & \multicolumn{2}{|c|}{ Platelet-lymphocyte ratio } & \multirow[t]{2}{*}{$p$ value } \\
\hline & & $<5(n=292)$ & $\geq 5(n=89)$ & & $<350(n=319)$ & $\geq 350(n=62)$ & \\
\hline $\operatorname{Sex}(\%)$ & & & & 0.32 & & & $0.07 *$ \\
\hline Male & $218(57.2)$ & $163(55.8)$ & $55(61.8)$ & & $176(55.2)$ & $42(67.7)$ & \\
\hline Female & $63(42.8)$ & $129(44.2)$ & $34(38.2)$ & & $143(44.8)$ & $20(32.3)$ & \\
\hline Age (mean, standard deviation) & $61.2 \pm 14.6$ & $60.4 \pm 14.3$ & $63.9 \pm 15.2$ & $0.045^{*}$ & $61.1 \pm 14.6$ & $61.6 \pm 14.7$ & 0.84 \\
\hline Tumor location(\%) & & & & 0.35 & & & 0.28 \\
\hline Antropylorus & $85(22.3)$ & $60(20.5)$ & $25(28.1)$ & & $71(22.2)$ & $14(22.6)$ & \\
\hline Gastric body & $215(56.4)$ & $168(57.5)$ & $47(52.8)$ & & $176(55.2)$ & $39(62.9)$ & \\
\hline Fundus & $72(18.9)$ & $58(19.9)$ & $14(15.7)$ & & $65(20.4)$ & $7(11.3)$ & \\
\hline Not reported & $9(2.4)$ & $6(2.1)$ & $3(3.4)$ & & $7(2.2)$ & $2(3.2)$ & \\
\hline Tumor differentiation (\%) & & & & 0.87 & & & 0.97 \\
\hline Well & $67(17.6)$ & $54(18.5)$ & $13(14.6)$ & & $57(17.9)$ & $10(16.1)$ & \\
\hline Moderated & $76(19.9)$ & $56(19.2)$ & $20(22.5)$ & & $65(20.4)$ & $11(17.8)$ & \\
\hline Poor & $117(30.7)$ & $89(30.5)$ & $28(31.5)$ & & $99(31.0)$ & $18(29.1)$ & \\
\hline Undifferentiated & $55(14.4)$ & $44(15.1)$ & $11(18.6)$ & & $45(14.1)$ & $10(16.1)$ & \\
\hline Unknown & $66(17.3)$ & $49(16.7)$ & $17(28.1)$ & & $53(16.6)$ & $13(20.9)$ & \\
\hline Lauren classification (\%) & & & & 0.79 & & & 0.28 \\
\hline Intestinal & $222(58.2)$ & $172(58.9)$ & $50(56.2)$ & & $192(60.2)$ & $29(46.8)$ & \\
\hline Diffuse & $159(41.8)$ & $120(41.1)$ & $39(43.8)$ & & $127(39.8)$ & $33(53.2)$ & \\
\hline TNM stage (\%) & & & & $0.006^{*}$ & & & $0.002 *$ \\
\hline I & $10(2.6)$ & $8(2.7)$ & $2(2.2)$ & & $9(2.8)$ & $1(1.6)$ & \\
\hline II & $100(26.3)$ & $81(27.7)$ & $19(21.3)$ & & $89(27.9)$ & $11(17.7)$ & \\
\hline III & $133(34.9)$ & $112(38.6)$ & $21(23.6)$ & & $119(37.3)$ & $14(22.6)$ & \\
\hline IV & $123(32.3)$ & $82(28.0)$ & $41(46.1)$ & & $91(28.5)$ & $32(51.6)$ & \\
\hline Unknown & $15(3.9)$ & $9(3.0)$ & $6(6.7)$ & & $11(3.5)$ & $4(6.5)$ & \\
\hline $\mathrm{ECOG}(\%)$ & & & & $0.031 *$ & & & $0.021 *$ \\
\hline 0 & $100(26.2)$ & $71(24.3)$ & $29(32.6)$ & & $84(26.3)$ & $16(25.8)$ & \\
\hline 1 & $217(57.0)$ & $179(61.3)$ & $38(42.7)$ & & $189(59.2)$ & $28(45.2)$ & \\
\hline 2 & $35(9.2)$ & $23(7.9)$ & $12(13.5)$ & & $27(8.5)$ & $8(12.9)$ & \\
\hline 3 & $21(5.5)$ & $13(4.5)$ & $8(9.0)$ & & $15(4.7)$ & $6(9.7)$ & \\
\hline 4 & $8(2.1)$ & $6(2.1)$ & $2(2.2)$ & & $4(1.3)$ & $4(6.5)$ & \\
\hline Surgery (\%) & & & & $0.006^{*}$ & & & $0.01 *$ \\
\hline Yes & $213(55.9)$ & $174(59.6)$ & $39(43.8)$ & & 187 (58.6) & $26(41.9)$ & \\
\hline No & $168(44.1)$ & $118(40.4)$ & $50(56.2)$ & & $132(41.4)$ & $36(58.1)$ & \\
\hline Chemotherapy (\%) & & & & $0.001 *$ & & & $0.036^{*}$ \\
\hline Yes & $206(54.1)$ & $172(58.9)$ & $34(38.2)$ & & $180(56.4)$ & $26(41.9)$ & \\
\hline No & $175(45.9)$ & $120(41.1)$ & $55(61.8)$ & & 139 (43.6) & $36(58.1)$ & \\
\hline
\end{tabular}

*Statistically significant at $p<0.05$

median survival time was 5.57 months (3.46-7.68) for patients with PLR $\geq 350$ and 17.07 months (95\% CI 11.7422.40) for patients with PLR less than 350 (HR 2.33; $95 \%$ CI 1.73-3.13, Log-rank test: $p<0.001)$.

Figure 2 shows the DFS probability of patients according to NLR and PLR categories. Events of recurrence or progression were more frequently observed in the high NLR and high PLR groups. Median DFS was 3.5 months (95\% CI 0.70-6.30) in patients with NLR $\geq 5$ and 15.43 months (95\% CI 10.05-20.81) in patients with NLR lower than 5 (HR 2.31; 95\% CI 1.78-3.00, Log-rank test: $p<0.001)$. Likewise, median DFS was 4.67 months (95\% CI 2.97-6.37) for patients with PLR $\geq 350$ versus 14.73 months (95\% CI 9.56-19-89) for patients with PLR less than 350 (HR 2.28; 95\% CI 1.70-3.06, Log-rank test: $p<0.001$ ).

The results of the univariate and multiple $\mathrm{COX}$ regression analyses for OS and DFS are presented in Tables 2 and 3, respectively. After adjustment for potential confounders, the NLR $\geq 5$ was independently associated with worse OS and 
Fig. 1 Overall survival probability (Kaplan-Meier method) according to the neutrophil-lymphocyte ratio (left) and platelet-lymphocyte ratio (right)

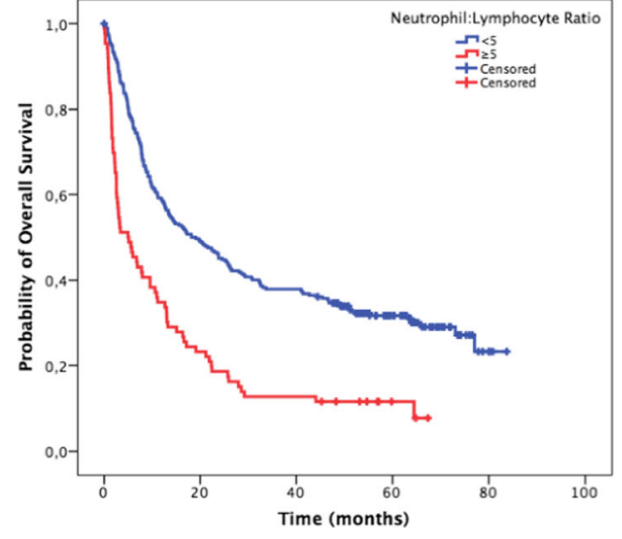

DFS. Also, TNM stage, ECOG performance status, and the absence of chemotherapy or surgery were associated with poor outcomes. The multivariate analyses did not reveal any statistically significant interaction between the NLR and the PLR (Tables 2 and 3).

\section{Discussion}

Inflammatory cells play an essential role in the pathogenesis and progression of cancer and are important determinants of patient's clinical outcome [13]. Neutrophils are thought to promote carcinogenesis by favoring angiogenesis, metastasis, and cell proliferation [14]. Cytotoxic lymphocytes are responsible of clearing tumor cells through recognition of tumorspecific antigens or tumor-associated antigens [13, 15]. Additionally, platelets adhere to tumor cells and facilitate their metastasis from the blood stream [16]. Therefore, the combination of increased number of circulating neutrophils, platelets, and lymphocytopenia could explain the poor outcomes of patients with high NLR and PLR.

In this study, we show that both the NLR and the PLR are prognostic factors in Hispanic gastric cancer patients from Costa Rica, a country with high incidence and mortality for this malignancy [3]. After covariate adjustment, only the NLR was independently associated with worse OS and DFS. Our findings are in accordance with previous reports in patients with solid tumors, including gastric cancer [11, 17-20]. Moreover, the majority of these studies assessing the role of NLR and PLR as prognostic biomarkers have been conducted in Asian populations, and almost no data from Latin-American countries are available. Recently, it has been determined that patients from Hispanic background tend to have poorer outcomes than Asian and White-non-Hispanic populations [8, 9]. We consider therefore that our results make an important contribution by evaluating the prognostic value of novel biological parameters in a geographical region where gastric adenocarcinoma represents a leading cause of death due to cancer [3-5].

Not all trials evaluating the prognostic usefulness of NLR and PLR in solid tumors come to the same conclusion and much of the heterogeneity found probably derives from different cutoff points used in each study. For example, in a recent metaanalysis of 33,432 patients with solid tumors [18], a range of cutoff values from 1.9 to 7.2 was associated with poor outcomes. In the present analysis, we decided to calculate the optimal NLR and PLR threshold based on the sequential approach suggested by Contal and O'Quigley [12]. A similar statistical methodology has been previously used by other authors [20] and it is preferred
Fig. 2 Disease-free survival probability (Kaplan-Meier method) according to the neutrophil-lymphocyte ratio (left) and platelet-lymphocyte ratio (right)
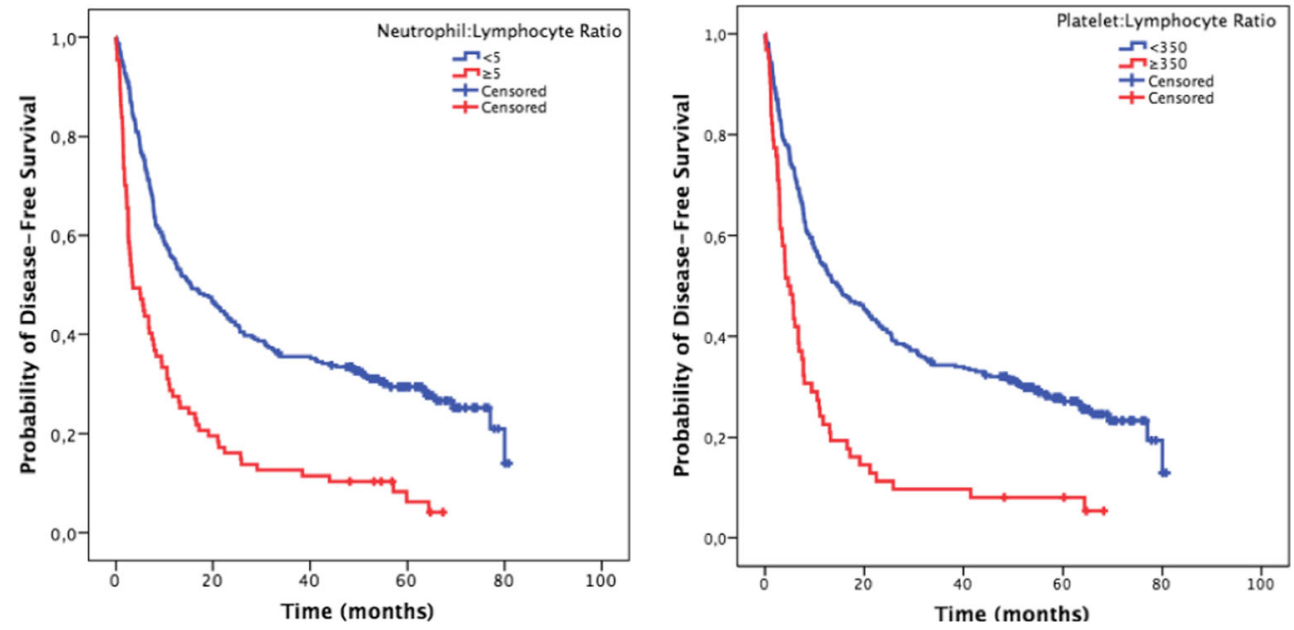
Table 2 Univariate and multivariate Cox regression analysis for overall survival

\begin{tabular}{|c|c|c|c|c|}
\hline Variable & $\begin{array}{l}\text { Crude hazard ratio } \\
\text { (95\% confidence interval) }\end{array}$ & $p$ value & $\begin{array}{l}\text { Adjusted hazard ratio } \\
\text { (95\% confidence interval) }\end{array}$ & $p$ value \\
\hline Sex & & 0.94 & & \\
\hline Female & 1.0 & & & \\
\hline Male & $0.94(0.74-1.19)$ & & & \\
\hline Age & & $0.062 *$ & & 0.45 \\
\hline$<60$ years & 1.0 & & 1.0 & \\
\hline$\geq 60$ years & $1.27(0.99-1.61)$ & & $1.11(0.85-1.45)$ & \\
\hline Tumor differentiation & & $0.001 *$ & & 0.25 \\
\hline Well & 1.0 & & 1.0 & \\
\hline Well moderated & $1.26(1.10-1.43)$ & & $1.06(0.96-1.17)$ & \\
\hline Poor & $2.52(2.20-2.86)$ & & $2.12(1.92-2.34)$ & \\
\hline Undifferentiated & $3.78(3.30-4.29)$ & & $3.18(2.88-3.51)$ & \\
\hline Lauren classification & & 0.99 & & \\
\hline Intestinal & 1.0 & & & \\
\hline Diffuse & $1.01(0.73-1.38)$ & & & \\
\hline TNM stage & & $<0.001 *$ & & $<0.001 *$ \\
\hline I & 1.0 & & 1.0 & \\
\hline II & $1.80(1.54-2.10)$ & & $1.44(1.23-1.70)$ & \\
\hline III & $3.60(3.08-4.20)$ & & $2.88(2.46-3.40)$ & \\
\hline IV & $5.40(4.62-6.30)$ & & $4.32(3.69-5.10)$ & \\
\hline ECOG & & $<0.001 *$ & & $0.004 *$ \\
\hline 0 & 1.0 & & 1.0 & \\
\hline 1 & $1.36(1.17-1.59)$ & & $1.24(1.07-1.44)$ & \\
\hline 2 & $2.72(2.34-3.18)$ & & $2.48(2.14-2.88)$ & \\
\hline 3 & $4.08(3.51-4.77)$ & & $3.72(3.21-4.32)$ & \\
\hline 4 & $5.44(4.68-6.36)$ & & $4.96(4.28-5.76)$ & \\
\hline Surgery & & $<0.001 *$ & & $<0.001 *$ \\
\hline No & 1.0 & & 1.0 & \\
\hline Yes & $0.27(0.21-0.35)$ & & $0.31(0.24-0.41)$ & \\
\hline Chemotherapy & & $0.014 *$ & & $<0.001 *$ \\
\hline No & 1.0 & & 1.0 & \\
\hline Yes & $0.74(0.58-0.94)$ & & $0.57(0.44-0.75)$ & \\
\hline NLR & & $<0.001^{*}$ & & $0.005 *$ \\
\hline$<5$ & 1.0 & & 1.0 & \\
\hline$\geq 5$ & $2.24(1.72-2.92)$ & & $1.59(1.15-2-28)$ & \\
\hline PLR & & $<0.001 *$ & & $0.079 *$ \\
\hline$<350$ & 1.0 & & 1.0 & \\
\hline$\geq 350$ & $2.33(1.73-3.13)$ & & $1.39(0.96-2.00)$ & \\
\hline NLR*PLR & & & 1.0 & 0.51 \\
\hline
\end{tabular}

**Statistically significant at $p<0.10$ over ROC curves, median values, or interquartile ranges because of its ability to control for multiple variables and time-to-event outcomes. Nevertheless, as other authors suggest, we must consider the NLR and PLR as continuous variables with increasing risk increments [21].

Although patients with high NLR were older, had more advanced disease, lower performance status and received more treatment (surgery or chemotherapy) than patients in the low NLR category, the multivariate analysis for OS and DFS showed that this index was not altered by these confounding variables. This implies that the NLR is a strong predictor for long-term outcomes and its incorporation into predictive nomograms is warranted, as recently showed by Choi and colleagues [22].

Gastric cancer prognosis is usually determined by pathological variables that are available after a surgical procedure or 
Table 3 Univariate and multivariate Cox regression analysis for disease-free survival

\begin{tabular}{|c|c|c|c|c|}
\hline Variable & $\begin{array}{l}\text { Crude hazard ratio } \\
\text { (95\% confidence interval) }\end{array}$ & $p$ value & $\begin{array}{l}\text { Adjusted hazard ratio } \\
\text { (95\% confidence interval) }\end{array}$ & $p$ value \\
\hline Sex & & 0.93 & & \\
\hline Female & 1.0 & & & \\
\hline Male & $0.99(0.78-1.25)$ & & & \\
\hline Age & & $0.071 *$ & & 0.45 \\
\hline$<60$ years & 1.0 & & 1.0 & \\
\hline$\geq 60$ years & $1.24(0.98-1.58)$ & & $1.17(0.90-1.52)$ & \\
\hline Tumor differentiation & & $0.002 *$ & & 0.25 \\
\hline Well & 1.0 & & 1.0 & \\
\hline Moderated & $1.22(1.07-1.39)$ & & $1.06(0.96-1.17)$ & \\
\hline Poor & $2.44(2.14-2.78)$ & & $2.12(1.92-2.34)$ & \\
\hline Undifferentiated & $3.66(3.21-4.17)$ & & $3.18(2.88-3.51)$ & \\
\hline Lauren classification & & 0.84 & & \\
\hline Intestinal & 1.0 & & & \\
\hline Diffuse & $0.97(0.70-1.33)$ & & & \\
\hline TNM stage & & $<0.001 *$ & & $<0.001 *$ \\
\hline I & 1.0 & & 1.0 & \\
\hline II & $1.82(1.56-2.13)$ & & $1.47(1.26-1.72)$ & \\
\hline III & $3.64(3.12-4.26)$ & & $2.94(2.52-3.54)$ & \\
\hline IV & $5.46(4.68-6.39)$ & & $3.41(3.78-5.16)$ & \\
\hline ECOG & & $<0.001 *$ & & $0.009 *$ \\
\hline 0 & 1.0 & & 1.0 & \\
\hline 1 & $1.34(1.15-1.55)$ & & $1.22(1.05-1.41)$ & \\
\hline 2 & $2.68(2.30-3.10)$ & & $2.44(2.10-2.82)$ & \\
\hline 3 & $3.92(3.45-4.65)$ & & $3.66(3.15-4.23)$ & \\
\hline 4 & $5.36(4.60-6.20)$ & & $4.88(4.20-5.64)$ & \\
\hline Surgery & & $<0.001 *$ & & $<0.001 *$ \\
\hline No & 1.0 & & 1.0 & \\
\hline Yes & $0.28(0.22-0.36)$ & & $0.32(0.25-0.42)$ & \\
\hline Chemotherapy & & $0.055^{*}$ & & $0.006^{*}$ \\
\hline No & 1.0 & & 1.0 & \\
\hline Yes & $0.79(0.63-1.00)$ & & $0.69(0.53-0.89)$ & \\
\hline NLR & & $<0.001 *$ & & $0.001 *$ \\
\hline$<5$ & 1.0 & & 1.0 & \\
\hline$\geq 5$ & $2.31(1.78-3.00)$ & & $1.97(1.44-2-47)$ & \\
\hline PLR & & $<0.001 *$ & & 0.24 \\
\hline$<350$ & 1.0 & & 1.0 & \\
\hline$\geq 350$ & $2.28(1.70-3.06)$ & & $1.13(0.78-1.64)$ & \\
\hline NLR*PLR & & & 1.0 & 0.64 \\
\hline
\end{tabular}

*Statistically significant at $p<0.10$ during disease staging. Conversely, the NLR and PLR can be easily calculated from hemogram results that are available in different settings with limited resources. Having inexpensive but reliable prognostic factors is particularly important in the context of gastric cancer since almost two-thirds of cases occur in developing countries $[1,2]$. The identification and validation of these sensitive biomarkers may help identify patients who may receive a different therapy and follow-up.
Our study has some limitations due to its retrospective design. However, it validates previous findings regarding the prognostic value of circulating neutrophils, platelets, and lymphocytes in patients with gastric cancer.

In conclusion, our findings show that the NLR is an independent prognostic factor for OS and DFS in Hispanic patients with gastric cancer regardless of their clinical stage, performance status, and treatment received. 
Further studies, however, should prospectively explore the prognostic value of these biomarkers in order to incorporate them into usual clinical practice.

\section{Compliance with Ethical Standards}

The study was approved by the Ethical Scientific Committee of the University of Costa Rica (no. 817-B2-371) and the Institutional Scientific Ethics Committee of the Caja Costarricense del Seguro Social (R013-SABI-00048). *The abstract of this manuscript was presented at the ESMO XX World Congress on Gastrointestinal Cancer 2018, Barcelona, Spain.

Conflict of Interest The authors declare that they have no conflict of interest.

\section{References}

1. International Agency for Research on Cancer. World Health Organization. Available at: http://globocan.iarc.fr/Pages/fact_ sheets cancer.aspx. Accessed on August 1 ${ }^{\text {st }}, 2017$.

2. Torre LA, Bray F, Siegel RL, Ferlay J, Lortet-Tieulent J, Jemal A. Global cancer statistics, 2012. CA Cancer J Clin. 2015;65:87-108.

3. Registro Nacional de Tumores. Ministerio de Salud, Gobierno de Costa Rica. Available at: https://www.ministeriodesalud.go.cr/ index.php/vigilancia-de-la-salud/estadisticas-y-bases-de-datos/ estadisticas/estadistica-de-cancer-registro-nacional-tumores?limit= 5. Accessed on May $26^{\text {th }}, 2018$.

4. Sierra M, Cueva P, Bravo LE, Forman D. Stomach cancer burden in central and South America. Cancer Epidemiol. 2016;44:S62-73.

5. Carioli G, La Vecchia C, Bertuccio P, et al. Cancer mortality predictions for 2017 in Latin America. Ann Oncol. 2017;28:2286-97.

6. Yao JC, Tseng JF, Worah S, Hess KR, Mansfield PF, Crane CH, et al. Clinicopathologic behavior of gastric adenocarcinoma in Hispanic patients: analysis of a single institution's experience over 15 years. J Clin Oncol. 2005;23:3094-103.

7. Rajabi B, Corral JC, Hakim N, Mulla ZD. Descriptive epidemiology of gastric adenocarcinoma in the state of Texas by ethnicity: Hispanic versus white non-Hispanic. Gastric Cancer. 2012;15:405-13.

8. Duma N, Sanchez LJ, Castro YS, Jennis AA, McCain D, Gutierrez $\mathrm{ME}$, et al. Gastric adenocarcinoma: clinicopathologic differences among Hispanics and non-Hispanic whites. A single Institution's experience over 14 years. Ann Gastroenterol. 2016;29:325-31.

9. Al-Rafaie WB, Tseng JF, Gay G, et al. The impact of ethnicity on the presentation and prognosis of patients with gastric adenocarcinoma. Results from the National Cancer Data Base. Cancer. 2008;113:461-9.

10. Ramos-Esquivel A, Rodriguez-Porras L, Porras J. Neutrophil to lymphocyte ration and platelet to lymphocyte ratio as prognostic factors in non-metastatic breast cancer patients from a Hispanic population. Breast Dis. 2017;37:1-6.

11. Wu J, Jiang $\mathrm{M}$, Qin $\mathrm{Y}$, et al. Single and combined use of neutrophil-lymphocyte ratio, platelet-lymphocyte ratio and carcinoembryonic antigen in diagnosing gastric cancer. Clin Chem Acta. 2018;481:20-4.

12. Contal C, O'Quigley J. An application of changepoint methods in studying the effect of age on survival in breast cancer. Comput Stat Data Anal. 1999;30:253-70.

13. Binnewies M, Roberts EW, Kersten K, Chan V, Fearon DF, Merad $\mathrm{M}$, et al. Understanding the tumor immune microenvironment (TIME) for effective therapy. Nat Med. 2018;24:541-50.

14. Mantovani A, Allavena P, Sica A, Balkwill F. Cancer-related inflammation. Nature. 2008;454:436-44.

15. Finn OJ. Cancer immunology. N Engl J Med. 2008;358:2704-15.

16. Gay L, Felding-Habermann B. Contribution of platelets to tumour metastasis. Nat Rev Cancer. 2011;11:123-34.

17. Zhang X, Zhang W, Feng L. Prognostic significance of neutrophil lymphocyte ratio in patients with gastric cancer: a meta-analysis. PLoS One. 2014;9:e111906.

18. Templeton AJ, McNamara MG, Seruga B, et al. Prognostic role of neutrophil-to-lymphocyte ratio in solid tumors: a systematic review and meta-analysis. J Natl Cancer Inst. 2014;106:dju 124.

19. XIn-Ji Z, Yong-Gang L, Xiao-Jun S, et al. The prognostic role of neutrophils to lymphocytes ratio and platelet count in gastric cancer: a meta-analysis. Int J Surg. 2015;21:84-91.

20. Lieto E, Galizia G, Auricchio A, Cardella F, Mabilia A, Basile N, et al. Preoperative neutrophil to lymphocyte ratio and lymphocyte to monocyte ratio are prognostic factors in gastric cancers undergoing surgery. J Gastrointest Surg. 2017;21:1764-74.

21. Altman DG, Lausen B, Sauerbrei W, Shumacher M. Danger of using optimal cutpoints in the evaluation of prognostic factors. J Natl Cancer Inst. 1994;86:829-35.

22. Choi JH, Suh YS, Choi Y, et al. Comprehensive analysis of the neutrophil-to-lymphocyte ratio for preoperative prognostic prediction nomogram in gastric cancer. World J Surg. 2018; https://doi. org/10.1007/s00268-018-4510-4. 